\title{
Aproximación cualitativa a la dinámica de una institución de educación superior desde la perspectiva de los docentes
}

\section{A qualitative approach to the dynamics of a higher education institution from the perspective of teachers}

\author{
Gustavo Enrique Cortez-Félix \\ Blanca Valenzuela \\ Manuela Guillen-Lúgigo \\ Universidad de Sonora
}

\begin{abstract}
Resumen
La presente investigación parte del interés de determinar qué procesos e incidentes de la dinámica de una universidad pública mexicana cobran relevancia según la percepción de los profesores universitarios, y a la vez se pretende indagar cómo estos eventos forman o modifican las identidades. Se utilizó un diseño cualitativo de teoría fundamentada, se entrevistó a profundidad a dieciocho profesores de diferentes programas; los discursos se analizaron en relación a descripciones, referentes, procesos, incidentes, dimensión de estructura y gobierno, dimensión política, dimensión laboral, dimensión académica, entre otras unidades. Como resultado se presenta un esquema que muestra los distintos itinerarios posibles según los relatos empíricos de los entrevistados, donde se tocan las dimensiones académica y laboral en cuanto a procesos relevantes en la trayectoria, asimismo se ofrece una descripción de la propia voz de los profesores. Se dedujo, por otra parte, que si bien la docencia es la actividad primordial realizada en la universidad, la dinámica institucional genera diferentes arreglos hostiles y tensiones en los docentes, al tener que responder a las presiones de las dimensiones políticas y laborales.

Palabras clave: Identidad docente, gobierno universitario, satisfacción laboral, academia, calidad educativa.
\end{abstract}

Nota del autor

Gustavo Enrique Cortez Félix, Departamento de Psicología, Universidad de Sonora; Blanca Valenzuela, Departamento de Psicología, Universidad de Sonora; Manuela Guillén Lúgigo, Departamento de Trabajo Social, Universidad de Sonora.

La correspondencia en relación con este artículo debe dirigirse a Gustavo Enrique Cortez Félix, Departamento de Psicología, Universidad de Sonora, Hermosillo, Sonora, 83000.

Dirección electrónica: gustavo_ecf@hotmail.com 


\begin{abstract}
This research departs from the interest to determine which processes and incidents of the dynamics of a Mexican public university become relevant as perceived by academics, as well as to explore how these events form or change identities. A qualitative grounded theory research design was made, in which deep interviews where done to eighteen teachers from different programs, the speeches were analyzed in relation to descriptions, references, processes, events, structural and government dimension, political dimension, labor dimension, academic dimension, among other units. As a result we display a diagram showing the different possible routes reported as experiences of the respondents where academic and professional dimensions touch on relevant processes in their pathways, also a description from the voice of the teachers is presented. Furthermore, although teaching is the primarily conducted activity at the university, the institutional dynamics generates different arrangements not without promoting frictions and tensions in teachers as they have to respond to the pressures of political and labor dimensions.
\end{abstract}

Keywords: Teacher identity, university governance, work satisfaction, academia, educational quality.

Las instituciones educativas tienen una dinámica por demás compleja que determina los procesos y resultados de los esfuerzos educativos. La calidad y excelencia educativa son resultado tanto de políticas y lineamientos como de esfuerzos humanos que se dan en el contexto diario de las prácticas educativas. Para Välimaa (2005), en los estudios de educación superior no se puede separar la investigación acerca de los cambios, mejora e innovación de la investigación sobre la estructura y tradiciones en el sistema.

Es de común acuerdo que, tal vez como en ninguna otra institución, el docente juega un papel sumamente importante, es fácil reafirmar esta posición si se entiende que de las capacidades y potencialidades para enseñar, investigar, crear arte y reproducir la cultura que tengan los docentes, dependerán las capacidades y potencialidades de las universidades. Ni los reglamentos ni la infraestructura hacen de la universidad lo que es ni le dan su relevancia social. Resulta, por lo tanto, que el docente es una fortaleza o amenaza para las instituciones educativas.

Es necesario, entonces, trabajar bajo entendimientos como el de Granja (2000), quien refiere que el campo de la educación, se debe analizar como un espacio de interacciones complejas entre agentes, prácticas, instituciones, regulaciones objetivadas, mecanismos de poder y legitimidad e intereses diversos en juego (Granja, 2000). Esto llevaría a dejar atrás visiones simplistas y reduccionistas acerca de las instituciones de educación superior como objeto de conocimiento y campo de estudio.

La perspectiva desde la cual se piensa la universidad y sobre la cual se plantea el problema que ocupa el presente trabajo, concibe a la institución como una realidad social compleja 
y dinámica, conformada por un componente formal y prescriptivo y otro informal donde juegan los actores educativos, que sin ser fijos, resumen un conjunto de disposiciones y prácticas libres no encuentran fragmentadas, sino que confluyen en múltiples configuraciones de la dinámica institucional.

Al mismo tiempo, es de interés entender al docente como una categoría singular $\mathrm{y}$ esto conlleva hacerse preguntas acerca de la construcción social e histórica de su identidad, su procedencia y su ubicación en la estructura social, su prestigio y diferencias que lo caracterizan; los docentes constituyen un conjunto social diferenciado cuya estructura y evolución sólo pueden percibirse si se les mira utilizando una serie de herramientas de observación generada en el interior de campos disciplinarios específicos (Tenti, 1984).

Siguiendo al autor, la universidad como organización provee determinados recursos, enmarca la acción con un conjunto de normas, cuenta con determinadas tradiciones y una cultura institucional específica, que influyen al momento de hacer cumplir los objetivos educativos; es relevante, por lo tanto, conocer el tipo de organización donde los docentes desarrollan su trabajo, con la posibilidad también de generar individual y colectivamente aquellos arreglos institucionales más favorables al logro de los objetivos que se propone con su desempeño. Para ello parece ser necesario entender a los docentes como parte del componente vivo $\mathrm{y}$, por la forma en que se colocan en el marco institucional como componente formal.

El objeto que se desea abordar forma parte de un proceso de construcción conceptual que considera el continuo entre los aspectos informales y los formales que definen la institución y que convergen para crear una dinámica institucional específica. De esta manera, se pretende que el foco de análisis sea el docente, al utilizar el concepto de identidad y de procesos e incidentes críticos como herramientas de dilucidación y quehacer investigativo.

En este sentido, se pretende retomar la identidad a partir de la referencia directa de los sujetos en relación con una pregunta básica: ¿Cuáles son los procesos e incidentes de la dinámica institucional y cómo forman o modifican las identidades de los profesores de la Unidad Regional Centro de la Universidad de Sonora (UNISON) en el periodo 1990-2012?

En el presente capítulo se exponen resultados parciales del estudio en relación con uno de sus objetivos específicos: identificar los procesos e incidentes críticos en la dinámica institucional desde una perspectiva cualitativa que retoma el discurso propio de los docentes. Se parte de una premisa: los sistemas educativos son particularmente complejos (Clark, 1987; Monclús, 2011; Rupérez, 1997); una verdadera apreciación acerca de los fenómenos que ocurren dentro de los sistemas educativos y la universidad, debe partir de la conceptualización del fenómeno educativo como un lugar de 
encuentro entre las posiciones estructurales objetivas y las disposiciones de los sujetos, de otra manera se cometerían errores de diagnóstico y se formularían políticas parcializadas (Tenti, 1984).

Para Zabalza (2002), conocer el marco legal de una institución, su estructura material y formal, es conocer poco; quedarse solamente en eso supone desconocer lo que realmente es la universidad y cómo es que ésta funciona: lo que la institución tiene de instituido y de instituyente, de tensiones y de acuerdos, los patrones de distribución de poder y de relaciones entre personas y funciones, etc.

Un importante desarrollo teórico en las ciencias sociales es el resurgimiento del interés en las instituciones. Aunque la definición varía según la disciplina, hay convergencia en la noción de la institución como reglas, normas de conducta y sus características de ejecución y vigilancia, que dan forma a la interacción humana. La educación superior, en tanto espacio social reglado y normado, tiene una rica variedad de posibilidades de comprensión bajo enfoques de análisis institucional (Dill, 2003).

De hecho, la universidad se considera una institución de las naciones-estado, dado que sirve a una función colectiva, define ciertos tipos de conocimientos y los legitima dentro de la sociedad, define principios culturales como cientificidad, racionalidad, leyes naturales, de ahí que termina siendo una estrategia para construir autoridad y legitimidad en la historia moderna (Meyer \& Rowan, 2006; Meyer \& Rowan, 1977; Meyer, Ramírez, Frank, \& Schofer, 2006).

Por otro lado, también se le considera como una serie de comunidades de interés derivadas de las profesiones académicas. Asimismo, la universidad como institución $\mathrm{u}$ organización incluye una serie de normas e incentivos de las diferentes disciplinas académicas, reglas y sanciones de la organización académica, políticas de regulación y mecanismos de vigilancia desde el gobierno institucional. Se diferencia, ampliamente, de otros tipos de instituciones estatales porque sus estructuras y contextos son muy específicos, nótese su organización en departamentos y facultades, formas de gobierno y relaciones entre la administración y la academia, la organización en disciplinas y la producción de conocimiento; condiciones que le otorgan características especiales (De Vries \& Ibarra, 2004).

Ordorika (1995) complementa la idea: se trata de organizaciones profesionales autónomas, con poca interdependencia entre los trabajadores, bajo condiciones de organizaciones extremadamente complejas, con tecnología de alta complejidad así como con un elevado nivel de incertidumbre y baja interdependencia. Siguiendo estas precisiones, se entenderá por institución el conjunto de prácticas organizadas estructuralmente $\mathrm{y}$ arraigadas en un espacio-tiempo, donde existen componentes que se refieren en específico al 
agente, mientras la institución como ente formal está organizada de forma estructural con base en la conjugación de ciertos recursos y reglas (Celis \& Guatame, 2003). La escuela se articularía por encadenamientos de acciones enmarcadas en un sistema de ordenamientos, que dan sentido y vida a la institución como tal. Para la escuela francesa de estudios institucionales (Lapassade, 1977), existen tres momentos básicos en las instituciones: lo instituido como lo que está dado y consolidado, lo instituyente como aquello que surge y tiende a instituir; y lo institucionalizado como la confluencia de los dos anteriores para crear a la institucionalidad. Se trata de un proceso continuo que hace converger momentos internos y externos en la institución.

Dado que las instituciones se definen por un compuesto de reglas que organiza la acción y que da estabilidad a la estructura, los mismos procesos para institucionalizar suelen surgir de las partes mejor constituidas en la organización, donde existen reglamentos explícitos y alta estabilidad, donde hay una historia y donde se conectan con más partes de la organización, de modo que un cambio afecta a otras partes de la subestructura (Zucker, 1987). En otras palabras, la universidad tiene una jerarquía formal con mecanismos de comunicación e interacción específicos entre las diferentes instancias $\mathrm{u}$ oficinas que tienen una tarea particular; estas mismas instancias crean sus jerarquías, reglamentos y mecanismos.

Siguiendo a Gewerc y Montero (1996), el componente formal del contexto organizativo tiene que ver con la razón social de la institución y las sub-instituciones dentro de la misma, con su política y divisiones de trabajo, con la manera en que se organiza y con los aspectos micropolíticos; mientras que las creencias, los conflictos y el juego de significados, que también contribuyen a configurar la organización de la universidad como institución educativa, tienen más relación con el componente informal.

El componente formal y estructural prescribe una actuación para cada uno de los agentes e instancias, además, la estructura-gobernanza y la dimensión política no solamente crean tendencias hacia ciertos comportamientos individuales y colectivos, por lo contrario colocan a los agentes en las estructuras de poder o las crean dando contexto a los juegos de poder, que desembocan en procesos relacionales. Aunado a esto, de los componentes formales surgen también los mecanismos que delimitan, además de la gobernanza, el funcionamiento hacia los objetivos institucionales, los cuales resultan en prácticas formales y no formales relacionadas con la academia, la ciencia y la cultura. Los elementos no formales y relacionales, surgidos de las interacciones e influencias al interior, forman parte del componente dinámico de las instituciones.

En otras palabras, se van estableciendo patrones de funcionamiento que tienen como base la estructura formal y legal de la institución, pero que la desbordan enriqueciéndose del juego relacional dentro de la propia institución, con sus componentes implícitos o explícitos. 
Estos juegos relacionales forman parte del componente no formal de las instituciones.

Dicha dialéctica entre los aspectos formales y no formales o relacionales, presentes en la dinámica institucional de las universidades, determina la actuación de los agentes y éstos aparecen como un inevitable marco de condiciones de la misma; también funcionan como un primer mecanismo para instaurar procedimientos, ritos y creencias a nivel institucional, por ejemplo: procedimientos de ingreso y promoción, ideas compartidas sobre los estudiantes, las teorías del aprendizaje y modelos de enseñanza en los modelos curriculares, etcétera.

La dinámica institucional sería, pues, el espacio donde los aspectos formales e informales se tocan y confluyen en procesos y hechos de diferente índole que marcan el funcionamiento real de la institución y en los cuales se puede apreciar claramente la interacción entre los agentes y las estructuras. Se denota, así, la forma en que los agentes se apropian y encarnan los recursos institucionales y, en el mismo sentido, la forma en que las estructuras organizan las prácticas escolares ideales para el orden institucional.

Para Zabalza (1995), existen cuatro criterios y condiciones de la dinámica institucional que son fundamentales para los centros educativos de calidad, éstos aluden a: los procesos de diseño de los procesos internos de las instituciones, a los productos educativos como logro de los objetivos institucionales, a los procesos para el logro de los resultados o productos $\mathrm{y}$, al propio desarrollo organizativo como un proceso diferenciado encaminado a la mejora de la organización escolar.

Por su parte, Musselin (2009), describe como resultado de la dinámica del trabajo académico desde una perspectiva sociológica a nivel mundial, la observación de fenómenos que tejen hilaciones con la división y regulación del trabajo entre docencia e investigación o gestión, misma que conlleva a un alejamiento e individualización de la planta docente; al mismo tiempo sucede una integración interna donde cada vez se constatan más esfuerzos por consolidar a las instituciones educativas con el fin de asegurar su calidad y prestigio.

Para Clark (1964), los resultados de la dinámica son los siguientes: reforzamiento de la autoridad y supervisión, asignación de labores y rotación de trabajadores académicos, estandarización de los procesos académicos, cambios en los métodos para la toma de decisiones y nuevas formas de incentivar la investigación y desarrollo. Por ejemplo, Bleiklie y Michelsen (2009) hacen alusión al juego entre los sindicatos y la administración en la definición de políticas de supervisión, asignación de tareas y promoción de los trabajadores académicos. Estos procesos e incidentes son básicos para la construcción de espacios comunes sobre los que se construye una identidad basada en los sujetos que la forman, sin embargo, no todos tienen la 
misma importancia, algunos hechos resaltan por ser críticos en la forma cómo influencian la construcción identitaria.

Siguiendo a Contreras, Monereo y Badia (2010), cuando el profesor se enfrenta a un incidente crítico negativo, su estabilidad personal cede terreno a la inseguridad, la inhibición y quizás a la agresividad, afectándole y poniendo en crisis su identidad, dado que no cuenta con los recursos para afrontar de manera oportuna y adecuada aquel incidente que lo cuestiona en lo que ha dicho, sentido, pensado y/o realizado. Para Bolívar, Fernández-Cruz y Molina (2005), si el docente se enfrenta a cambios drásticos en el contexto socioeducativo se puede dar una crisis de identidad, que es también parte intrínseca de la construcción de esa identidad. Esta crisis es la expresión de un modo de salvaguardar la propia identidad profesional que el cuerpo académico percibe como seriamente amenazada; la crisis identitaria docente se da en la medida que, con la formación recibida y con la práctica docente que ha formado el saber profesional, no se puede responder a las nuevas exigencias y funciones, a los cambios sociales y del alumnado.

\section{Método}

La metodología del estudio corresponde a un diseño de teoría fundamentada, procedimiento propuesto por Glaser y Strauss (1967). El método y sus procesos de análisis son inductivos y buscan generar una teoría explicativa para un fenómeno social específico mediante técnicas de interpretación y codificación. Los procedimientos para la investigación son específicos y su seguimiento resulta en el alcance de los objetivos y metas de investigación por medio de la reducción de los discursos, su contrastación y comparación, apegado a un proceso de codificación constante con base en interpretaciones y códigos previamente diseñados según las categorías y variables de interés.

Se utiliza un sistema de códigos fundamentados en los indicadores y conceptos relevantes para este estudio: descripciones, referentes, procesos, incidentes, dimensión de estructura y gobierno, dimensión política, dimensión laboral, dimensión académica, efectos, entre otros. Se realiza, además, un proceso de codificación abierta para determinar si aparecen categorías no contempladas con anterioridad y que cobren relevancia explicativa en función de las preguntas y objetivos de investigación. Se presenta, entonces, la sistematización de todos los discursos utilizando un conjunto de figuras o esquemas que se refieren a los conceptos relevantes y a las relaciones entre dichos conceptos, respaldados en una serie de unidades de significado que ejemplifica los resultados obtenidos.

Para la selección de los participantes se utiliza el proceso de muestreo teórico, donde se consideraron como deseables y necesarias la representatividad, la diversidad y la capacidad de respuesta para buscar la saturación teórica. El muestreo está determinado por los objetivos 
$\mathrm{y}$ preguntas de investigación y la muestra se puede modificar si es necesario. Se consideran acabados el proceso y la recolección cuando se alcanza la saturación teórica, es decir, cuando no se encuentra nueva información sobre las categorías conceptuales y cuando los datos obtenidos explican suficientemente cada categoría.

Fueron seleccionados 18 profesores, tres por cada división, bajo el criterio de contar con una antigüedad mayor o igual a 20 años, lo cual representa haber ingresado a la docencia en la Universidad de Sonora (UNISON) durante el año 1991 o anterior, para ello se cuenta con la relación de profesores y sus antigüedades hasta el año 2011. Esta decisión de corte metodológico se ha asumido en tanto se considera que es imprescindible acotar en espacio y tiempo el objeto de estudio.

Se considera el año de 1991 como fecha de corte porque marcaría el proceso de implementación de la Ley Orgánica 4 y, por consiguiente, la instauración de una nueva dinámica institucional, de tal manera que los profesores integrados a la institución desde 1991 o antes, tienen la posibilidad de relatar y referir aspectos relevantes a nuestro objeto de estudio. Esto se logra por medio de un sondeo de referencias, utilizando pues un muestro de juicio por medio de la técnica de bola de nieve. De estos 18 profesores, fue seleccionado a uno de cada división para realizar una segunda entrevista a fondo y así esclarecer los cabos sueltos en las entrevistas generales, además de dar mayor profundidad a la información previamente recabada.

De los 18 participantes en la estrategia cualitativa, diez de ellos fueron hombres y ocho mujeres. Como se ha referido, se entrevistó tres profesores por cada división. La edad media fue de 56.4 y la antigüedad promedio de 32.7. Seis profesores tienen grado de licenciatura, ocho de maestría y cuatro de doctorado. El total de profesores ha participado en el Programa de Estímulos al Desempeño del Personal Docente (PEDPD) en diversas ocasiones, actualmente, solo nueve de ellos participaron en la última convocatoria, asimismo, diez cuentan con Perfil PROMEP (Programa para el Mejoramiento del Profesorado) y uno es miembro del Sistema Nacional de Investigadores (SNI).

Los entrevistados indican que la actividad a la cual le dedican mayor tiempo es la docencia $\mathrm{y}$ en tres casos, por igual a la docencia e investigación. En el historial de los participantes se encuentra que los mismos han realizado tareas administrativas, desempeñado cargos directivos, como coordinadores de programa, jefe de departamento, miembros de los consejos divisionales y académicos, así como haber participado en comisiones de revisión y evaluación de los programas.

\section{Resultados}

Para identificar los procesos o incidentes críticos, además de una serie de preguntas específicas sobre el tema, se indagó en las 
historias personales de los entrevistados dentro de la institución y en su percepción sobre las trayectorias de los docentes, con esto se ha elaborado un diagrama que concentra los diferentes caminos genéricos de los profesores (ver figura 1).

Como se muestra en la parte superior de la figura, la carrera en la universidad para los docentes tiene inicio de dos formas: ya sea por haber estudiado en la UNISON o bien en otra institución. En el primer caso se identificaron dos caminos para adentrarse en la dinámica institucional fuera del rol de estudiante, ya sea por apoyar en la docencia a algún profesor, ayudar en un laboratorio o incluso apoyar en investigación; el otro camino es involucrarse en la política universitaria en grupos estudiantiles o en figuras del gobierno universitario. De esta manera se obtiene el ingreso como docente y una contratación por horas o con una plaza de tiempo completo en muchos casos, una vez dentro, generalmente, se da un cambio en las

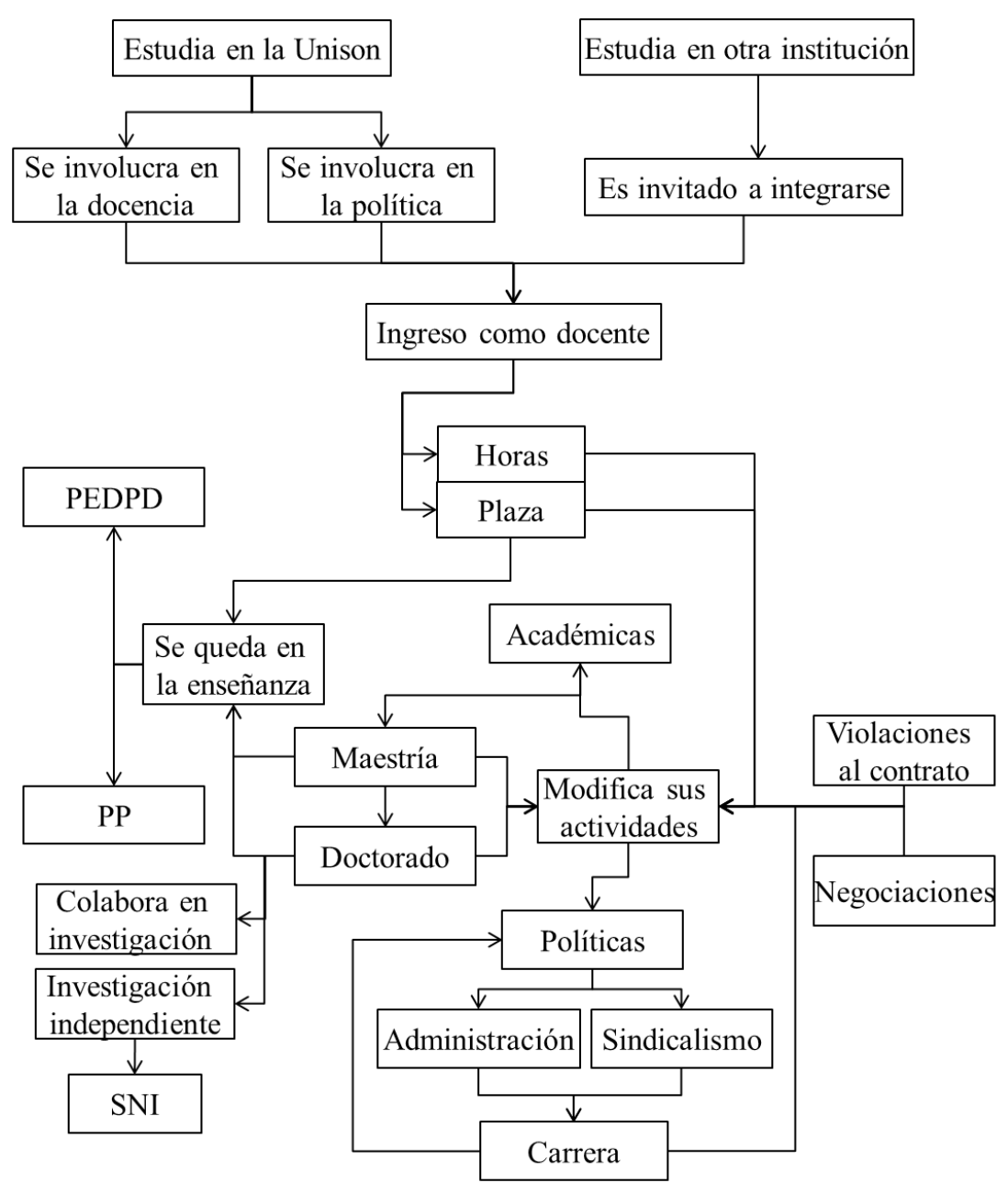

Figura 1. Trayectoria general de los docentes. 
actividades o bien, la actividad posterior solo contempla la enseñanza.

La modificación de las actividades puede ser en términos académicos o políticos, en el primer caso lo usual ha sido optar por los estudios de posgrado, que no implican necesariamente cursar el doctorado, hay docentes que después de la maestría se dedican en exclusivo a la enseñanza pudiendo también colaborar en la investigación, estos docentes tienden a participar en el PEDPD y el Perfil PROMEP como forma de superación y medio para obtener otros ingresos complementarios al salario, bien hay quienes deciden no participar o la enseñanza es una tarea secundaria, puesto que a la vez se dedican a trabajar fuera de la institución. Quienes siguen hacia los estudios de doctorado terminan dejando la investigación y dedicándose solo a la enseñanza (incluso abandonan los estudios de doctorado), colaborando en investigación con otros investigadores o consolidándose como investigadores con producción independiente y original llegando en muchos casos a ingresar al SNI.

En el segundo caso, cuando las actividades son primordialmente políticas, se encuentran dos caminos: la política dentro del organigrama administrativo de la institución o en la organización sindical. Se identifican carreras completas dentro de los aparatos administrativos de la universidad y del sindicato, que llevan en muchos casos a acceder a diferentes posiciones políticas y de gestión por largos periodos de tiempo, "profesionalizando" a estos docentes, quienes en gran medida dejan la enseñanza e investigación.

Las violaciones al contrato y las negociaciones salariales y contractuales aparecen como hechos que llevan a modificar las actividades políticas, por ejemplo, incitan a participar activamente en el sindicato para obtener una plaza, subsanar un problema específico o acceder a nuevos beneficios. Los dos caminos no son excluyentes, por ejemplo, después de consolidar una carrera académica, los docentes se adentran al terreno político universitario con el respaldo de su reputación o bien utilizan los espacios y beneficios alcanzados políticamente para consolidar o complementar su carrera académica.

La trayectoria genérica obtenida de las entrevistas permite identificar una serie de hechos y procesos relevantes en la dinámica institucional y en la vida propia de los docentes, éstos se apegan fielmente a las dimensiones que se tratan en la investigación. Para profundizar, se examinaron los posibles elementos de cada dimensión de la dinámica institucional (a saber: estructura y gobierno, política, laboral y académica), que a partir de los discursos identificados tienen relevancia para los participantes; se obtuvo una serie de procesos e incidentes que se resume en categorías generales a las cuales se asocia una o varias propiedades que les dan la cualidad de tener un efecto.

En la figura 2 se presenta la dimensión de estructura y gobierno, donde se establecieron cuatro categorías que se consideraron con 
alta relevancia a partir de los discursos de los participantes encuestados. Los elementos destacados son la ley orgánica, el cambio de autoridades, la participación y el sistema de administración; las líneas continuas representan relaciones entre las categorías y las líneas punteadas asocian las propiedades a las categorías.

Sobre la participación se comenta sobre la inexistencia de consultas a la comunidad académica, o bien, se trata de una simulación; en todo caso se consulta a pequeños grupos dentro de la esfera política de las autoridades a cargo. El participante 16 retoma estos señalamientos: "diría que depende del tipo de cosas que se van a decidir, puedes elegir tener la opinión de los líderes académicos o de los grupos de profesores, y cuando los requieres la gente se junta y toma decisiones, pero casi siempre que nos requieren es porque las decisiones ya se tomaron entre unos cuantos y todo esto de la participación es una simulación".
Que el sistema de administración sea excluyente alude además, a la escasa participación, reflejada como resultado de procesos poco democráticos donde, la toma de decisiones se da sin sustento académico, a discreción o placer de las autoridades. El participante 17 cree que "hace falta noción en la administración de lo que realmente significa cambiar, lo que sucede es que lo que nos dirigen no tienen una noción sobre el cambio educativo y la cosa fundamental que son los humanos".

Sobre los cambios de autoridades el participante 6 refiere que no hay cambios debido a que "el sistema por el cual se determina quién va a tener un puesto relevante, sea jefe, director, o vicerrector, tal vez no tanto con el rector, es un sistema perverso que crea una condición deplorable en la planeación institucional, los cambios de autoridades están programados para que todos sigan la línea del edificio principal y entonces no hay diversidad en las estructuras de gobierno, no importan los cambios, todo sigue siendo como el de máximo quiera".

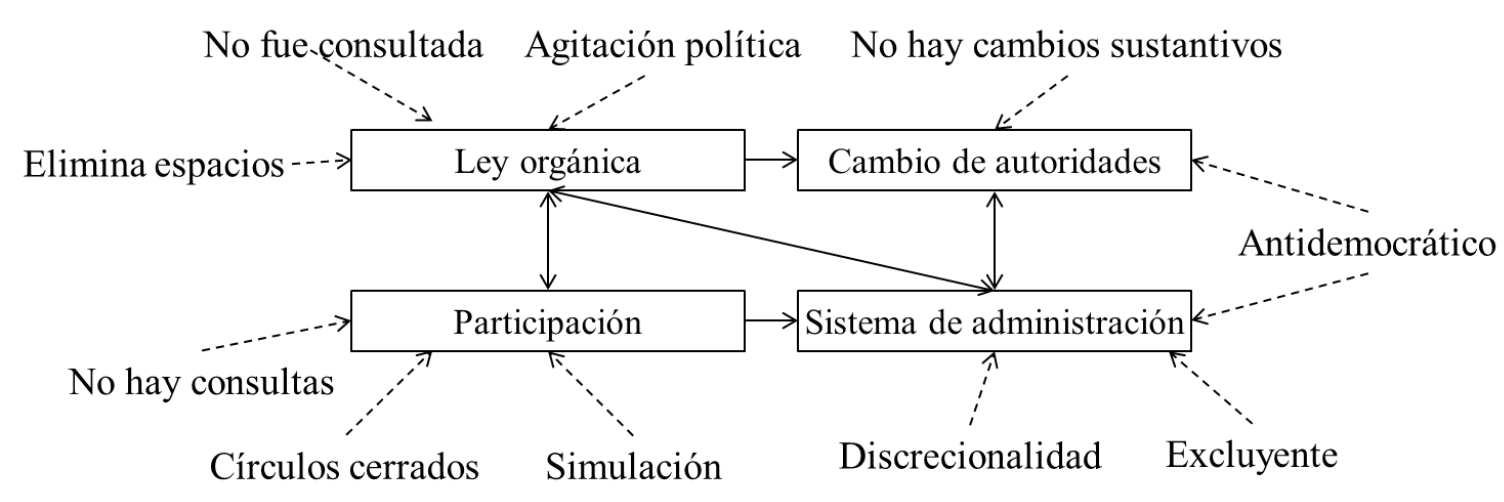

Figura 2. Categorías relevantes en la dimensión de estructura y gobierno. 
Por último, el entrevistado 1 en su discusión sobre la ley dice: "la imposición de la Ley 4, yo creo que eso fue un golpe duro a la universidad en cuanto a la estructura de gobierno que tenía antes, yo creo que ahí se perdió mucho de la participación de los profesores y los alumnos en la toma de decisiones, un hecho muy fuerte para la gente que ya teníamos tiempo aquí", refiriéndose a los espacios de participación que se cerraron.

Los elementos relevantes en la dimensión política se presentan en la figura 3, éstos son las elecciones de autoridades, el manejo académico, manejo administrativo, sindicalismo y negociaciones del contrato.

En relación con la categoría de las elecciones de autoridades, el participante 5 indica que "cuando fue la elección del jefe de nuestro departamento se formaron los grupos y cada quien por su lado, o sea, son tus colegas de todos los días, pero luego llega el momento de apoyar a alguien y todo el mundo se da por la espalda".
Sobre el manejo académico se dijo que "los docentes al estar frente al ejercicio fundamental de la universidad que es el conocimiento, son más conscientes que los administrativos de lo que eso implica, el administrativo está sumido en su lógica del poder y del control, es necesario, pero no al grado que lo han practicado".

Las menciones sobre el manejo administrativo también versan en el mismo sentido; además de resaltar la nula rendición de cuentas y la toma de decisiones a discreción de las autoridades, un entrevistado comenta: "los movimientos de cambios de jefe de departamento, de jefe de división, toda esa burocracia política afecta la imagen al exterior, internamente nos afecta mucho en la visión y la misión de la universidad, los cambios de autoridades que llegan con otros equipos y que dañan al buen funcionamiento de la universidad, entonces eso está dañando el buen funcionamiento de la universidad y repercute en la función del académico" (participante 3).

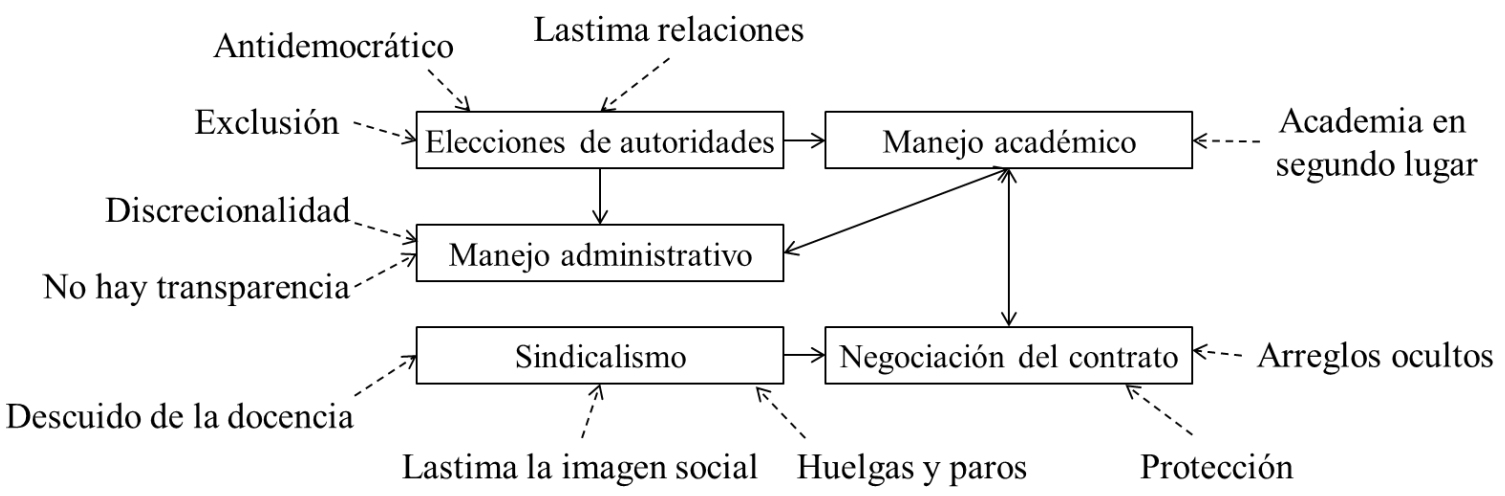

Figura 3. Categorías relevantes en la dimensión política. 
Sobre la cuestión sindical y las negociaciones del contrato se presenta una mención que abarca las dos categorías y varias propiedades: "hay cosas muy estresantes y tiempos estresantes, cuando se viene la huelga y no vas a tiempo con el programa te estresas mucho, o cuando vas bien en el programa, pero la universidad te pide que trabajes en otras cosas y te empiezas a atrasar, no te alcanza el tiempo para una cosa o la otra, pero bueno, cuando las cosas no funcionan entre la administración y los trabajadores o nosotros los profesores solamente nos queda ir a la huelga porque es la forma más importante para hacer valer lo que merecemos, pero también sufrimos el desgaste aunque muchos estudiantes no lo ven, no estamos contentos con dejar temas pendientes por ir a la huelga".

El tema de sindicalismo y las negociaciones del contrato tienen claramente un efecto por naturaleza en la materia o dimensión laboral, por ejemplo: “desgraciadamente a veces nos vemos involucrados en problemas internos que manchan esa visión y misión, principalmente son los movimientos de huelga, las disputas de los contratos colectivos de trabajo de los sindicatos, no tanto las huelgas sino el manejo político de los contratos colectivos, se ha dividido a los docentes a veces, $y$ eso trae problemas a veces, $y$ el sistema educativo se ha demeritado tanto que ahora en términos educativos no se encuentra en manos de los maestros, sino en los líderes sindicales... y eso trae muchas consecuencias, pero luego las cosas no son unilaterales, los quieren hacer así, pero conocemos el contrato colectivo de trabajo y no nos dejamos que quieran violarlo" (participante 7).

Con referencia a lo anterior, sobre la dimensión laboral, se presenta la figura 4 donde aparecen los elementos de violaciones al contrato, contrato colectivo, burocracia, reglamentos, concurso y posición académica.

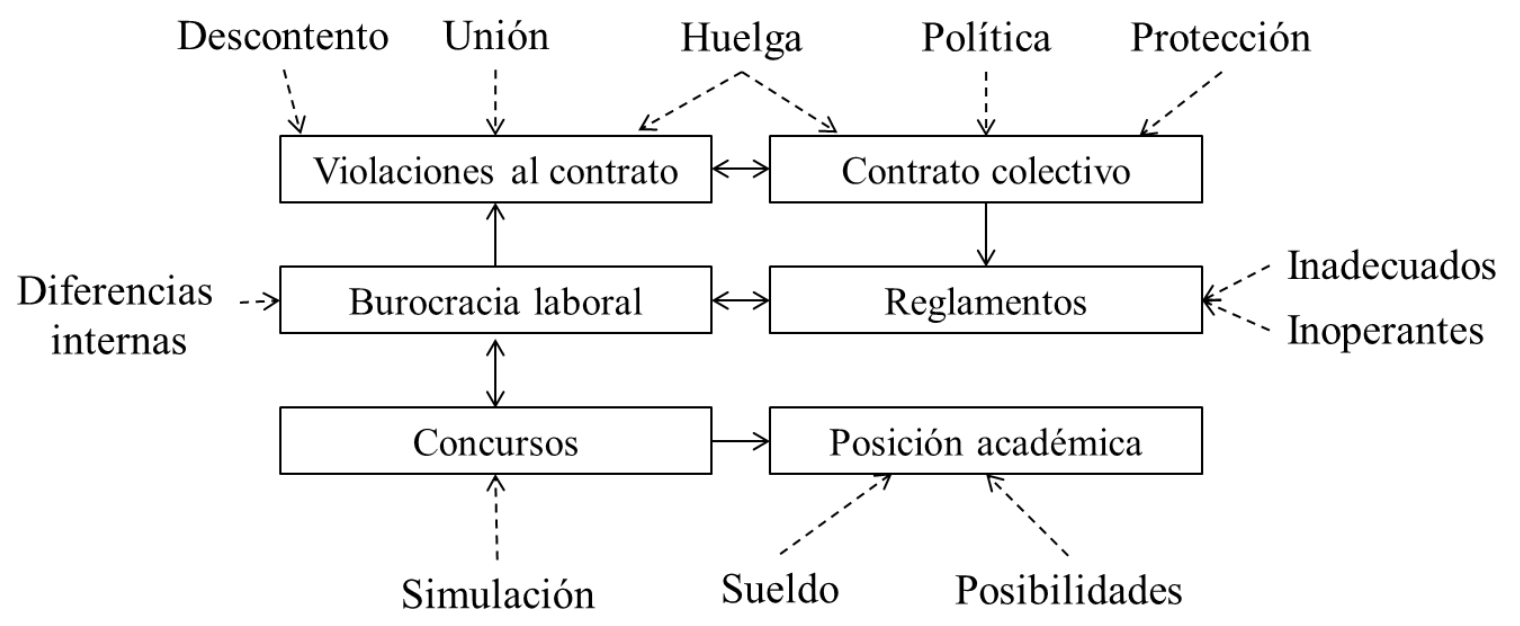

Figura 4. Categorías relevantes en la dimensión laboral. 
En relación íntima con el contrato y el sindicalismo, aparece la categoría de burocracia laboral, en la cual confluyen los procesos y mecanismos para acceder a las prestaciones y condiciones laborales en lo general, no implica necesariamente una organización burocrática. Al respecto, el participante 11 expresa que "las cuestiones administrativas nos afectan mucho, de la parte administrativa que se hizo aliada de la parte política externa, que está buscando mermar ese tipo de prestaciones, hay una dirección pésima en ese sentido, un lugar que premia más que el saber, la docilidad, siendo universitario es deplorable, levantar la mano a favor de, en cambio de todo lo que quieras no es como debe de ser, faltan cosas, los mecanismos no aseguran que las prestaciones sean suficientes en cuanto al monto, y en algunos rubros habría que agregar, serían adecuadas si alcanzaran, pero no alcanza, en el seguro de gastos médicos mayores uno tiene que pagar pero no todo".

Sobre los reglamentos o más bien la falta de ellos en materia laboral, el participante 1 menciona: "tenemos un problema en el caso de la jubilación, si la universidad no tiene algo reglamentado o estructurado pues estamos muy mal, podemos tener todo mientras tanto, pero si no existe un esquema que permita la seguridad a futuro de esos sujetos, quiere decir que las cosas no están muy bien".

Para finalizar la dimensión laboral, se toca el tema de los concursos y la contratación, el participante 4 dice que un problema "se trata del ingreso, las convocatorias salen cerradas y no entran los mejores, entran los de antigüedad y más derechos creados en la UNISON... por ejemplo, para el ingreso está bien marcado el procedimiento y los requisitos, el problema es la relatividad de los puntajes y procedimientos, así como uno puede falsear papeles para el PROMEP o el SNI, también pudiera hacerlos para ingresar, y bueno más que eso es que realmente no se revisa la pertinencia de lo que se presenta y entra el que sea". La posición académica implica tanto el tipo de contratación, el nivel de cualificación (grado) y desempeño, ya que la posición facilita o dificulta acceder a espacios y oportunidades: "resulta que los investigadores también participan en el PROMEP, y los requisitos que necesitan para esa beca, son fáciles para el investigador y los requisitos para entrar al SNI son difíciles para el docente" (participante 14).

En la figura 5 se trata la dimensión académica con los elementos de cambio en la tarea docente, cambios del contexto de la docencia, trabajo colegiado, retroalimentación, participación en programas y calidad educativa.

El cambio del modelo educativo se encuentra dentro de los cambios en el contexto de la docencia, en el caso particular de la institución, el participante 18 expresa que "el nuevo modelo curricular, se centra en el conocimiento del estudiante, en el aprender a aprender, es puro conocimiento, el estudiante no les encuentra ningún valor, no es significativo, tenemos que cambiar competencias y habilidades para que sientan que lo aprendido es útil, tiene un valor 


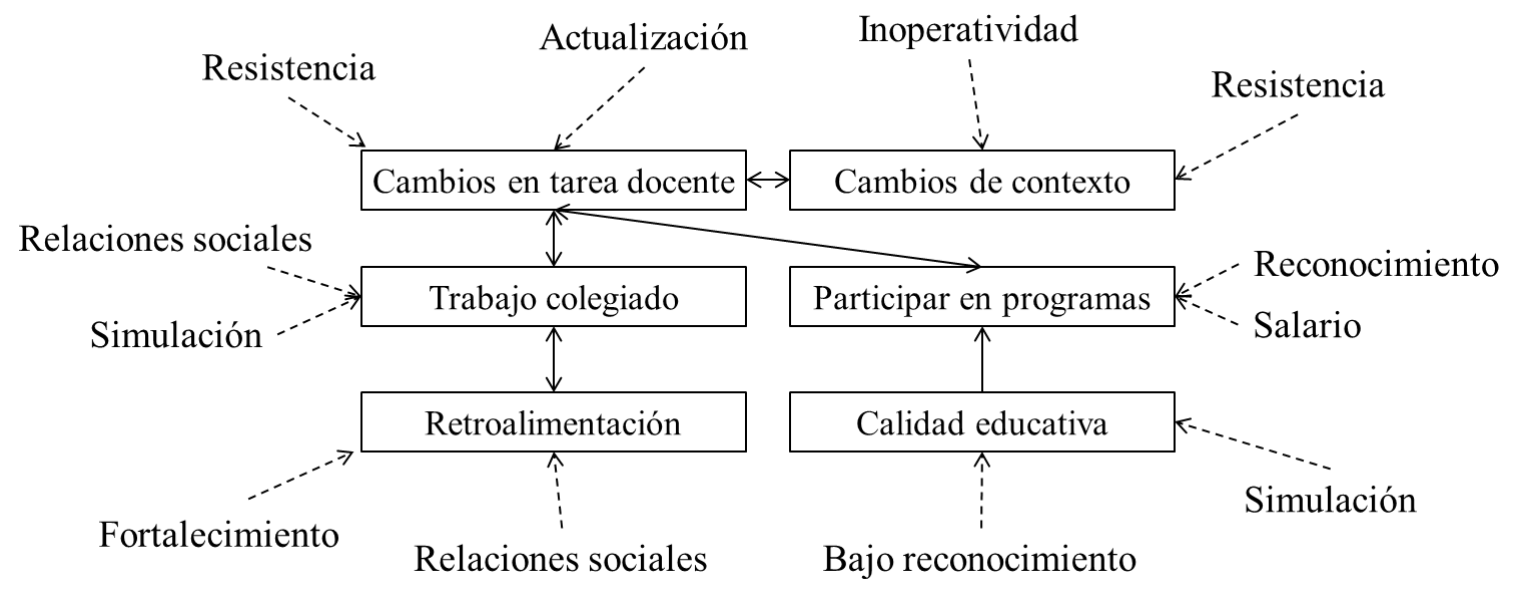

Figura 5. Categorías relevantes en la dimensión académica.

significativo para ellos, el profesor tiene que cambiar su forma de dar la clase, de ser el centro, para transmitir el conocimiento, irlo soltado", se encuentra aquí también una relación directa con el cambio en la tarea docente.

El trabajo colegiado se considera una simulación cuando las decisiones se consultan y se pretenden tomar en equipo, pero al final la opinión de los docentes no es tomada en cuenta o bien, ya se ha tomado una decisión. En este sentido, anteriormente se ha presentado un segmento de discurso sobre la participación que ilustra este tipo de expresiones, sin embargo, vale la pena notar expresiones positivas como: "en el modelo curricular, no te voy a decir que no tuvimos problemas y todavía los tenemos, el problema radica en que era una sola licenciatura con cuatro opciones, música, canto, plásticas, danza y teatro... nos fuimos por el trabajo intuitivo y por cómo funcionaba el plan viejo, no es por competencias, hacemos un buen trabajo colegiado, los maestros entramos a ver las clases de los otros para saber cómo complementarnos, así reflexionamos sobre lo que estamos haciendo, lo que hicimos el semestre pasado" (participante 13), manifiestamente se toca además el cambio del contexto de la docencia en cuanto al cambio del modelo curricular.

En correspondencia y acerca de la retroalimentación, otro entrevistado refiere que hay trabajo en equipo y comunicación entre profesores para mejorar en la docencia al poner énfasis en los estudiantes: "hemos hecho ejercicios de autocrítica entre profesores y eso ha permitido saber hablar de ellos (de los problemas de la docencia), en grupo o en seminarios se da poco, pero sí lo hacemos, con los alumnos todos los días, hay más con los alumnos que con los colegas, las preguntas de los alumnos, las actitudes, los comentarios, el nivel de los estudiantes, esa retroalimentación, aprendemos de los alumnos, un curso nunca 
lo damos de la misma manera y hay variables que influyen para bien o para mal, aunque los alumnos sepan menos que el profesor, es el valor de la retroalimentación" (participante 16).

Sobre la participación en programas esta unidad de significado ha sido seleccionada especialmenteporquesubrayalas dospropiedades principales que se le han asociado: "anualmente nos evalúan para la beca del desempeñó docente, entre más produzcas tienes mejor nivel, y cada tres el Perfil PROMEP, si escribes y produces pues tienes un reconocimiento, para los doctores el SNI, son tres logros en los que tienes que hacer cosas y estarse actualizando, son cosas de estatus y económicas" (participante 6). Asimismo, sobre la simulación y el bajo reconocimiento o atención a los docentes en la evaluación de la calidad, el participante 7 toca las dos propiedades: "la acreditación no muestra calidad porque es una simulación, se fijan en el aspecto físico y en los espacios, que si tenemos buenos espacios, buenos centros de cómputo y bibliotecas, en lo académico y en la capacidad y fortaleza de los docentes es más simulación".

Para finalizar esta sección, la figura 6 resume las cuatro dimensiones de la dinámica institucional con sus distintos elementos e incorpora líneas punteadas que representan las relaciones entre éstos cuando pertenecen a diferentes dimensiones.

Claramente, hay una relación entre los cambios de autoridades y las elecciones de los mismos, sin embargo, la relación no se marca solo por su naturaleza, sino porque los referentes de antidemocracia, exclusión y poca participación son numerosos. Además, con la materia estructural y la política hay una relación similar entre el sistema de administración y el manejo administrativo. Lo político se relaciona, en principio, con lo académico por

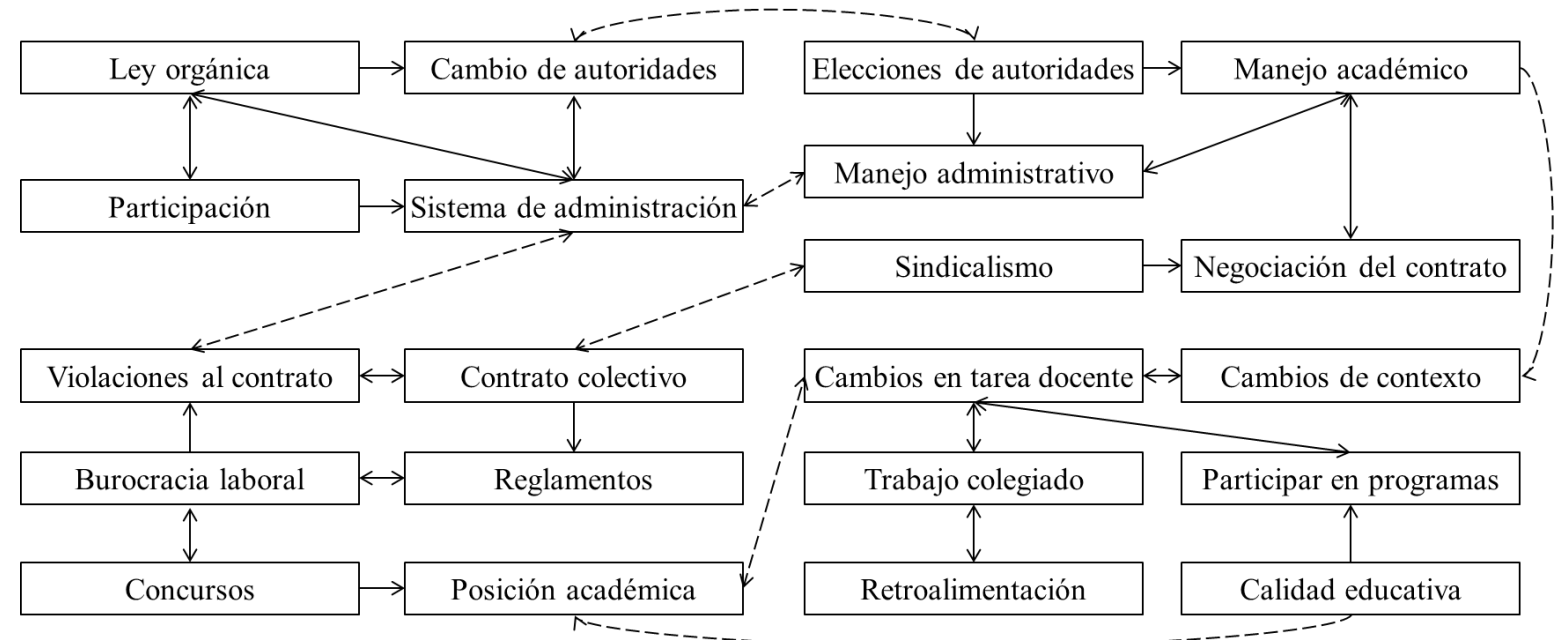

Figura 6. Relaciones entre las categorías relevantes de las dimensiones de la dinámica institucional. 
la influencia del manejo académico en los cambios del contexto de la docencia. En tal sentido el participante 14 opina: "la universidad debe cambiar en su conjunto, no nada más en las prácticas, los contenidos y los modelos educativos, estas cosas que son dirigidas desde arriba como si por mandato se fueran a dar".

La calidad educativa se relacionó con la participación en los programas de reconocimiento y fomento a la calidad académica, se refirió que la participación representa indicadores considerados para determinar la calidad de los cuerpos académicos. Se relaciona también con los cambios en la tarea del docente, porque éstos introducen demandas que modifican su actividad, como publicar y titular estudiantes. La relación entre el contrato colectivo y el sindicalismo también es inherente y se puede considerar que tienen cualidades similares. Las violaciones al contrato se relacionan con el sistema de administración como lo ejemplifica el participante 12: "luego no hay escapatoria, la administración decide y aunque sabe que está afectando nuestros derechos laborales, pues hace lo que le plazca, promete y no cumple, por eso hay tanta insistencia cada año en las negociaciones, por eso dicen los estudiantes que siempre pedimos lo mismo y que nos empecinamos buscando más y más cuando es la administración la que no cumple".

\section{Discusión}

Los resultados de la exploración nos permiten observar que si bien la docencia es la actividad principal en la universidad, la dinámica institucional genera diferentes arreglos que acarrean tensiones a los docentes, quienes tienen que responder a las presiones de las dimensiones políticas y laborales, principalmente. Tal situación concuerda con la tendencia mundial que se presenta dentro de las universidades en relación con los cambios en las condiciones de trabajo, en gran medida debido a las presiones externas (El-Khawas, 2009).

Así como los docentes padecen diferentes presiones a las cuales tienen que responder, se espera atender la docencia al aumentar constantemente el número de estudiantes, se espera la investigación y publicación constante, se espera que el docente participe en diferentes comisiones y reuniones, que se encargue de la extensión y trate con el sector público y privado a nombre de la misma institución; debe de atender una alta burocracia, competir por mejores sueldos, no se siente tomado en cuenta en la toma de decisiones y lo desmotiva la simulación que observa en diferentes procesos trascendentes para la calidad administrativa y propiamente educativa.

Ya adelantaba Clark (1980) que la cultura académica se ha diferenciado hacia dentro, en 
respuesta a la complejidad de las dinámicas institucionales, hablando ahora de culturas disciplinares, profesionales, organizacionales, del sistema, de la administración y de la investigación. Los resultados, entonces, confirman una situación generalizada: los docentes tienen diferentes afiliaciones y formas de envolverse en la dinámica institucional, sufren de una alta tensión entre los valores profesionales y las expectativas del sistema burocrático, demandan autonomía en el trabajo dada su experiencia y habilidad en su campo y prefieren la evaluación y regulación entre pares (Baldridge, Curtis, Ecker, \& Riley, 1974; Ecker \& Baldridge, 1973).

Los estudios de Henkel (2005) muestran que la disciplina y la autonomía-libertad son dos factores clave para la formación de la identidad académica. La libertad refiere a la autoorganización y autodeterminación de la docencia universitaria como factor que da seguridad, significado y autoestima a los profesores. No obstante, se observa una tendencia preocupante que reduce precisamente la autonomía: ante los nuevos requerimientos del docente, éste experimenta una falta de tiempo que afecta directamente la calidad de la docencia y la satisfacción de los profesores (Barnett, 2009).

\section{Referencias}

Baldridge, J. V., Curtis, D. V., Ecker, G. P., \& Riley, G. L. (1974). Alternative Models of Governance in Higher Education. Research and Development Memorandum, 129.
Barnett, R. (2009). Being An Academic In A Time-Impoverished Age. En A. Amaral, I. Bleiklie \& C. Musselin (Eds.), From Governance to Identity (Vol. 24, pp. 7-17). Netherlands: Springer.

Bleiklie, I., \& Michelsen, S. (2009). The University As Enterprise And Academic CoDetermination. En A. Amaral, I. Bleiklie \& C. Musselin (Eds.), From Governance to Identity (Vol. 24, pp. 57-78). Netherlands: Springer.

Bolívar, A., Fernández-Cruz, M., \& Molina, E. (2005). Investigar la identidad profesional del profesorado: Una triangulación secuencial. Forum Qualitative Sozialforschung/Forum Qualitative Social Research, 6(1).

Celis, J. E., \& Guatame, P. C. (2003). La dinámica institucional, la sociabilidad y las prácticas escolares: Un acercamiento metodológico desde la sociología. Revista Colombiana de Sociología, 21, 207-230.

Clark, B. R. (1964). Social Trends and Educational Organization. 59th Annual Meeting of the American Sociological Association. Recuperado de http://eric. ed.gov/?id=ED025210

Clark, B. R. (1980). Academic culture. New Haven, Conn: Higher Education Research Group, Institution for Social and Policy Studies, Yale University.

Clark, B. R. (1987). The Problem of Complexity in Modern Higher Education. Working Paper 9. 
Contreras, C., Monereo, C., \& Badia, A. (2010). Explorando en la identidad: ¿Cómo enfrentan los docentes universitarios los incidentes críticos que ocurren en las aulas de formación de futuros profesores? Estudios Pedagógicos, 36(2), 63-81.

De Vries, W., \& Ibarra, E. (2004). La gestión de la Universidad: Interrogantes y problemas en busca de respuestas. Revista Mexicana de Investigación Educativa, 9(22), 575-558.

Dill, D. D. (2003). An Institutional Perspective on Higher Education Policy: The Case of Academic Quality Assurance. En J. C. Smart (Ed.), Higher Education: Handbook of Theory and Research (Vol. 18, pp. 669-699). Netherlands: Springer.

Ecker, G. P., \& Baldridge, J. V. (1973). Academic Politics, Morale, and Involvement: Preliminary Findings of the Stanford Project on Academic Governance. Staford, CA: Stanford Center for Research and Development in Teaching.

El-Khawas, E. (2009). Emerging Academic Identities: A New Research And Policy Agenda. En A. Amaral, I. Bleiklie \& C. Musselin (Eds.), From Governance to Identity (Vol. 24, pp. 31-44). Netherlands: Springer.

Gewerc, A., \& Montero, L. (1996). Profesores universitarios: Contextos organizativos y desarrollo profesional. Enseñanza, 14, 65-79.

Glaser, B. G., \& Strauss, A. L. (1967). The Discovery of Grounded Theory: Strategies for Qualitative Research. NY: Aldine de Gruyter.

Granja, J. (2000). Configuración de conocimientos sobre la educación: Aspectos sociohistóricos y epistémicos. Colección Pedagógica Universitaria, 32-33, 55-71.

Henkel, M. (2005). Academic identity and autonomy in a changing policy environment. Higher Education, 49(1), 155-176. doi: 10.1007/s10734-004-2919-1

Lapassade, G. (1977). Grupos, organizaciones e instituciones: La transformación de la burocracia. Barcelona: Gedisa.

Meyer, H. D., \& Rowan, B. (2006). The new institutionalism in education. NY: State University of New York Press.

Meyer, J., \& Rowan, B. (1977). Institutionalized Organizations: Formal Structure as Myth and Ceremony. The American Journal of Sociology, 83(2), 340-363. doi: citeulikearticle-id:661594

Meyer, J. W., Ramírez, F. O., Frank, D. J., \& Schofer, E. (2006). Higher Education as an Institution. CDDRL Working Papers, 57.

Monclús, A. (2011). La educación entre la complejidad y la organización. Granada: Universitario.

Musselin, C. (2009). Towards A Sociology Of Academic Work. En A. Amaral, I. Bleiklie \& C. Musselin (Eds.), From Governance to Identity (Vol. 24, pp. 47-56). Netherlands: Springer.

Ordorika, I. (1995). Organización, gobierno y liderazgo universitario: Una base conceptual 
para el análisis del cambio en la educación superior. Universidades, 10, 39-48.

Rupérez, F. L. (1997). Complejidad y Educación. Revista Española de Pedagogía, 206, 103-112. Tenti, E. (1984). La interacción maestro-alumno: Discusión sociológica. Revista Mexicana de Sociología, 46(1), 161-174.

Välimaa, J. (2005). Social Dynamics of Higher Education Reforms: The Case of Finland. En Å. Gornitzka, M. Kogan \& A. Amaral (Eds.), Reform and Change in Higher Education (Vol. 8, pp. 245-267). Netherlands: Springer. Zabalza, M. A. (1995). Dinámica institucional de las escuelas y calidad de la educación. Cuadernos de Sección. Educación 8, 53-72. Recuperado de http://www.euskomedia.org/ PDFAnlt/ikas/08/08053072.pdf

Zabalza, M. A. (2002). La enseñanza universitaria: El escenario y sus protagonistas. Madrid: Narcea.

Zucker, L. G. (1987). Institutional Theories of Organization. Annual Review of Sociology, 13(1), 443-464. doi:10.1146/annurev. so.13.080187.002303 